\title{
Pulmonary Bacterial Communities in Surgically Resected Noncystic Fibrosis Bronchiectasis Lungs Are Similar to Those in Cystic Fibrosis
}

\author{
Heather Maughan,, 2 Kristopher S. Cunningham, ${ }^{3}$ Pauline W. Wang, ${ }^{1,2}$ Yu Zhang, \\ Marcelo Cypel, ${ }^{4}$ Cecilia Chaparro, ${ }^{5}$ D. Elizabeth Tullis, ${ }^{5}$ Thomas K. Waddell, ${ }^{4}$ \\ Shaf Keshavjee, ${ }^{4}$ Mingyao Liu, ${ }^{4}$ David S. Guttman, ${ }^{1,2}$ and David M. Hwang ${ }^{3,4,6}$ \\ ${ }^{1}$ Centre for the Analysis of Genome Evolution \& Function, University of Toronto, Toronto, ON, Canada M5S 3B2 \\ ${ }^{2}$ Department of Cell \& Systems Biology, University of Toronto, Toronto, ON, Canada M5S 3G5 \\ ${ }^{3}$ Department of Laboratory Medicine \& Pathobiology, University of Toronto, Toronto, ON, Canada M5S $1 A 8$ \\ ${ }^{4}$ Latner Thoracic Surgery Research Laboratories, University Health Network, Toronto, ON, Canada \\ ${ }^{5}$ Division of Respirology, St. Michael's Hospital and University of Toronto, Toronto, ON, Canada \\ ${ }^{6}$ Department of Pathology, Toronto General Hospital, University Health Network, Room 11E423, 200 Elizabeth Street, \\ Toronto, ON, Canada M5G 2C4
}

Correspondence should be addressed to David M. Hwang, david.hwang@uhn.on.ca

Received 15 July 2011; Accepted 27 October 2011

Academic Editor: Stefano Centanni

Copyright (C) 2012 Heather Maughan et al. This is an open access article distributed under the Creative Commons Attribution License, which permits unrestricted use, distribution, and reproduction in any medium, provided the original work is properly cited.

\begin{abstract}
Background. Recurrent bacterial infections play a key role in the pathogenesis of bronchiectasis, but conventional microbiologic methods may fail to identify pathogens in many cases. We characterized and compared the pulmonary bacterial communities of cystic fibrosis (CF) and non-CF bronchiectasis patients using a culture-independent molecular approach. Methods. Bacterial $16 \mathrm{~S}$ rRNA gene libraries were constructed from lung tissue of 10 non-CF bronchiectasis and 21 CF patients, followed by DNA sequencing of isolates from each library. Community characteristics were analyzed and compared between the two groups. Results. A wide range of bacterial diversity was detected in both groups, with between 1 and 21 bacterial taxa found in each patient. Pseudomonas was the most common genus in both groups, comprising $49 \%$ of sequences detected and dominating numerically in 13 patients. Although Pseudomonas appeared to be dominant more often in CF patients than in non-CF patients, analysis of entire bacterial communities did not identify significant differences between these two groups. Conclusions. Our data indicate significant diversity in the pulmonary bacterial community of both CF and non-CF bronchiectasis patients and suggest that this community is similar in surgically resected lungs of CF and non-CF bronchiectasis patients.
\end{abstract}

\section{Introduction}

Bronchiectasis is a heterogenous condition characterized by chronic infection/inflammation and irreversible abnormal dilatation of the airways. Generally classified into cystic fibrosis (CF) and non-CF disease, bronchiectasis was associated with a high mortality rate in the preantibiotic era [1]. While the outcome of bronchiectasis, both $\mathrm{CF}$ and non-CF, has improved significantly, non-CF bronchiectasis (hereafter referred to simply as bronchiectasis) nevertheless remains a significant cause of respiratory morbidity that necessitates surgical resection and even lung transplantation in some patients.

While the cause of CF has been pinpointed to mutations in a single gene, non-CF bronchiectasis may be associated with a wide range of different conditions including ciliary dysmotility syndromes, immune deficiencies, and chronic obstructive pulmonary disease (COPD) and, in many cases, is idiopathic [2]. The common underlying pathogenic mechanism, however, appears to be recurrent airway infection and 
inflammation resulting in scarring and permanent dilatation of the airways.

Given the centrality of airway infection in the pathogenesis of bronchiectasis, a number of studies have endeavored to identify bacterial pathogens present in these patients, using conventional microbiologic methods [3-11]. Such studies have typically found the most common pathogens to be nontypable Haemophilus influenzae and Pseudomonas aeruginosa, followed by a handful of others including Moraxella catarrhalis, Streptococcus pneumoniae, Staphylococcus aureus, and coliforms. Nontuberculous mycobacteria have also been reported with variable frequency. Such studies suggest that, despite seemingly similar pathophysiologic mechanisms, there may be differences in the bacterial species infecting $\mathrm{CF}$ and non-CF bronchiectasis patients. For example, while $H$. influenzae is the most common pathogen in adult nonCF bronchiectasis (approximately $40 \%$ of cases), it is far less prevalent in adult CF patients $[1,12]$. On the other hand, $P$. aeruginosa is far more commonly isolated among adult CF patients than from bronchiectasis patients (approximately $80 \%$ versus $18 \%$, resp.) $[1,12]$. Further, the Burkholderia cepacia complex, a group of organisms associated with significantly impaired outcome in CF patients, infects bronchiectasis patients quite infrequently in comparison to CF patients [13]. These observations suggest that despite similar changes in the disease morphology within the airways, other factors and mechanisms may be at play in determining what organisms are able to infect each group of patients.

These studies, however, almost certainly underestimate the bacterial diversity of the lungs in patients with bronchiectasis, given that $30-40 \%$ of sputum samples may fail to grow pathogenic bacteria in conventional culture, despite being of good quality and purulent [2]. Such studies, therefore, may underestimate the extent of diversity and variation within the pulmonary bacterial flora associated with bronchiectasis. Application of the methods and strategies of an emerging field of study known as metagenomics may help address these issues. Metagenomics is the study of communities of organisms using genetic material taken directly from their natural environment [14]. Metagenomics' power comes from its ability to assess community structure and dynamics without needing first to isolate and propagate organisms in the laboratory. Free of the constraints and limitations imposed by culture-based methods, it provides a semiquantitative assessment of community diversity. Communities can be studied without any prior knowledge of their composition and without the need for specific selective conditions, by isolating and characterizing DNA directly from the metagenome-that is, the combined genomes of all species present in the specimen of interest-bypassing the need for laboratory isolation and cultivation. The potential power of metagenomics to investigate questions related to health and disease has been increasingly recognized in recent years, as evidenced, for example, by the recent launches of the International Human Microbiome Consortium (http://www.human-microbiome.org/), the Human Microbiome Project (HMP), and other similar initiatives around the world $[15,16]$.
A growing number of studies using culture-independent molecular strategies for detection of bacterial species have demonstrated striking diversity of the pulmonary bacterial community in CF patients [17-27]. However, few if any similar studies have been done to characterize pulmonary bacterial communities in bronchiectasis. We, therefore, endeavored to profile the diversity of the pulmonary bacterial community in patients with bronchiectasis by sequencing bacterial $16 \mathrm{~S}$ rRNA gene libraries constructed using surgically excised lung tissue from 10 patients with bronchiectasis; we compared these communities to those seen in 21 patients with CF.

\section{Methods}

2.1. Patients and Sample Collection. Protocols for specimen collection, storage, and use were approved by the University Health Network (UHN) Research Ethics Board. Under aseptic conditions, tissue specimens were collected from patients with an established diagnosis of either CF or non-CF bronchiectasis undergoing surgical resection (pneumonectomy) or lung transplantation at the Toronto General Hospital between 1998 and 2007. Peripheral lung tissues were collected under sterile conditions intraoperatively, flash frozen in liquid nitrogen, and stored at $-80^{\circ} \mathrm{C}$. Specimens from 10 patients with non-CF bronchiectasis and 21 randomly selected CF patients were analyzed (Table 1). All specimen processing was performed blinded to patient data. Clinical data were extracted from electronic patient records at UHN.

2.2. DNA Extraction and $16 S$ rRNA Gene Amplification and Sequencing. Genomic DNA was extracted using the Direct PCR DNA Extraction System (Viagen Biotech, Los Angeles, CA). PCR reactions and negative controls were prepared in a separate room from where amplifications were conducted. Amplification of $16 \mathrm{~S}$ rRNA was performed using the $8 \mathrm{~F}\left(5^{\prime}\right.$-AGAGTTTGATCCTGGCTCAG) and 806R $\left(5^{\prime}-\right.$ GGACTACCAGGGTATCTAAT) PCR primers, as follows: 1 cycle $\left(95^{\circ} \mathrm{C}, 5 \mathrm{~min}\right) ; 30$ cycles $\left(95^{\circ} \mathrm{C}, 30 \mathrm{sec} ; 55^{\circ} \mathrm{C}, 30 \mathrm{sec}\right.$; $\left.72^{\circ} \mathrm{C}, 30 \mathrm{sec}\right) ; 1$ cycle $\left(72^{\circ} \mathrm{C}, 8 \mathrm{~min}\right)$. Amplicon libraries for each specimen were constructed using the TA cloning kit (Invitrogen, Carlsbad, CA) and plated on LB media. Plasmids from individual isolates were purified using the Pure Link Quick Plasmid Miniprep kit (Invitrogen). DNA sequencing was performed on an Applied Biosystems 3130XL sequencer (Analytical Genetics Technology Centre, Toronto) on up to 48 isolates initially, followed by preliminary assessment of diversity using BLAST [28]. Specimens containing more than one taxon were subjected to additional sequencing. Chimeras were identified by BLAST analysis of end sequences and excluded from analysis.

2.3. Data Analysis. Sequences were analyzed using the Qiime community analysis pipeline [29]. Sequences were clustered into operational taxonomic units (OTUs) using a 97\% identity threshold. One sequence representing the most abundant from each OTU was chosen for taxonomic classification 
using the RDP classifier [30]. Taxonomy classifications with a support level greater than or equal to 0.7 were retained; this resulted in not all sequences being classified to the most resolved (i.e., genus) level. All whole bacterial community comparisons included all sequences regardless of the level to which their taxonomy was assigned; however, for the identification and analysis of numerically dominant taxa, we chose only those OTUs classified to the genus level since this has the most functional information. OTU taxonomy classifications were used to construct the OTU table used in rarefaction analysis, heatmap construction, and distance calculations. Rarefaction analysis was performed in Qiime [29], using 100 replicate samplings of the sequences for subsequent estimation of alpha diversity. Calculation of UniFrac metrics [31] and principal components analyses were done in Qiime [29]. Clustering for heatmap presentation was performed in $\mathrm{R}[32]$.

\section{Results}

3.1. Bacterial Community Diversity of the Lungs in Patients with Bronchiectasis and CF. In total, 955 16S rRNA gene sequences were obtained from lung tissue specimens of 10 patients with bronchiectasis (mean $96 \pm 29$ (sd) per patient) and 1387 16S rRNA gene sequences from lung tissue specimens of 21 patients with CF (mean $66 \pm 24$ (sd) sequences per patient). Etiologies underlying the diagnoses of bronchiectasis included postinfectious (4 patients), immune dysfunction (3 patients), chronic obstructive pulmonary disease ( 1 patient), and idiopathic ( 2 patients). Taxonomic classification identified 37 unique operational taxonomic units (OTUs) representing 20 genera in bronchiectasis patients and 51 unique OTUs representing 29 genera in CF patients, with $16.7 \pm 2.9$ OTUs representing $9.1 \pm 1.7$ genera per patient in bronchiectasis and $11.5 \pm 6.0$ OTUs representing $6.8 \pm 3.5$ genera per patient in CF (range 1-21) (Table 1). These OTUs represented 4 phyla, Proteobacteria being most numerous in both groups (Table 2), and included many taxa not typically reported as constituents of the respiratory tract microflora (Table 3 ).

Pseudomonas was the most prevalent genus overall, with a total of 9 OTUs being detected in 30 of 31 patients, including all 10 bronchiectasis patients, and accounting for $49 \%$ of all sequences (Table 3). However, Pseudomonas was the numerically predominant genus (i.e., the genus represented by the largest number of $16 \mathrm{~S}$ rRNA sequences) in only 13 patients, with various other taxa predominating in the other 18 patients. Pseudomonas was numerically predominant more frequently in CF patients (11/21 patients) than in bronchiectasis (2/10 patients), although this difference was not statistically significant $(P=0.18,2$-sided Fisher's Exact Test). Sequences from other genera known to colonize adult CF patients were also detected, including Stenotrophomonas and Staphylococcus in both patient groups. Haemophilus was detected in only 2 patients with CF and 1 patient with bronchiectasis. Interestingly, Burkholderia sequences were detected at low levels in 4 patients with bronchiectasis.

We used rarefaction analysis to determine whether the number of sequences we obtained from each patient

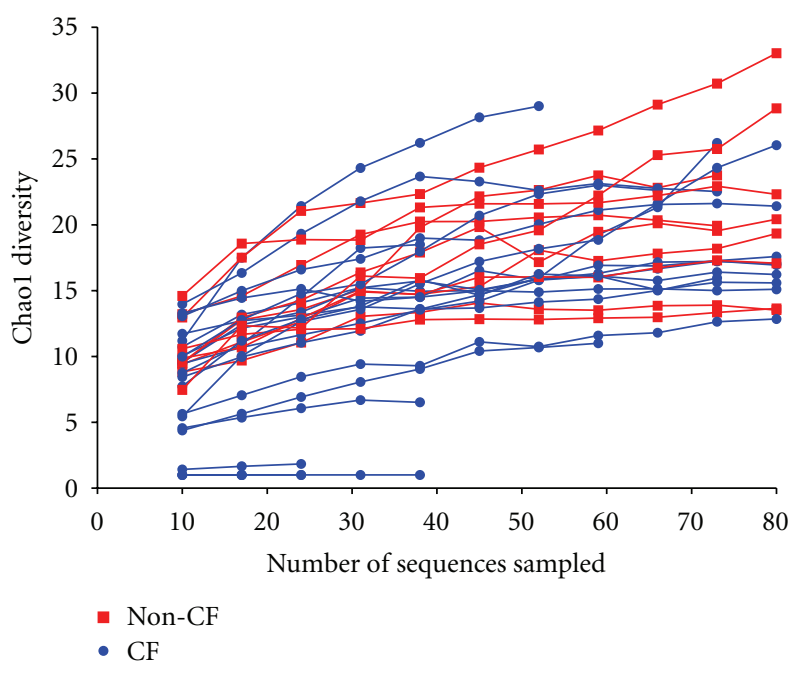

FIGURE 1: Rarefaction curves estimating the effect of sampling on Chao 1 diversity measurements. The sequence data were resampled 100 times in Qiime [29], and, for each sample, the Chaol diversity measurement was estimated. Red lines indicate bronchiectasis patients, whereas blue lines indicate cystic fibrosis (CF) patients.

was a sufficient sample of the sequence diversity present. Rarefaction analysis works by repeated sampling of the data; with each of these samples, the OTU diversity is calculated and plotted to determine whether the observed diversity increases with more sampling. If the sampling saturates the diversity present, then the curve will plateau, whereas if there is additional diversity yet to be discovered, the curve will continue to rise. Considering the relatively low number of sequences we sampled for each patient, most of the rarefaction curves indicate sufficient sampling of the diversity present (Figure 1). Although there are a few patient curves that are undersampled (i.e., that continue to increase), these are not restricted to bronchiectasis or CF patients alone, indicating that any potential differences in bronchiectasis versus CF community structure are unlikely to be due to insufficient sampling.

3.2. Comparison of Pulmonary Bacterial Communities in Bronchiectasis and CF. We compared the bacterial communities between bronchiectasis and CF patients in three ways: clustering of community profiles, clustering of community distances, and principal components analysis. Community profiles were clustered and are represented in the heatmap shown in Figure 2. Although there is a small cluster of bronchiectasis-derived communities (B188, B150, B366, and B193), those not clustered together are interspersed with the CF communities.

The heatmap clustering is informative for comparing patients based on their community profiles but does not consider sequence differences within or between OTUs. Therefore, we also compared the sequence divergence in these community profiles within a phylogenetic framework by calculating the normalized weighted UniFrac distance [31] 


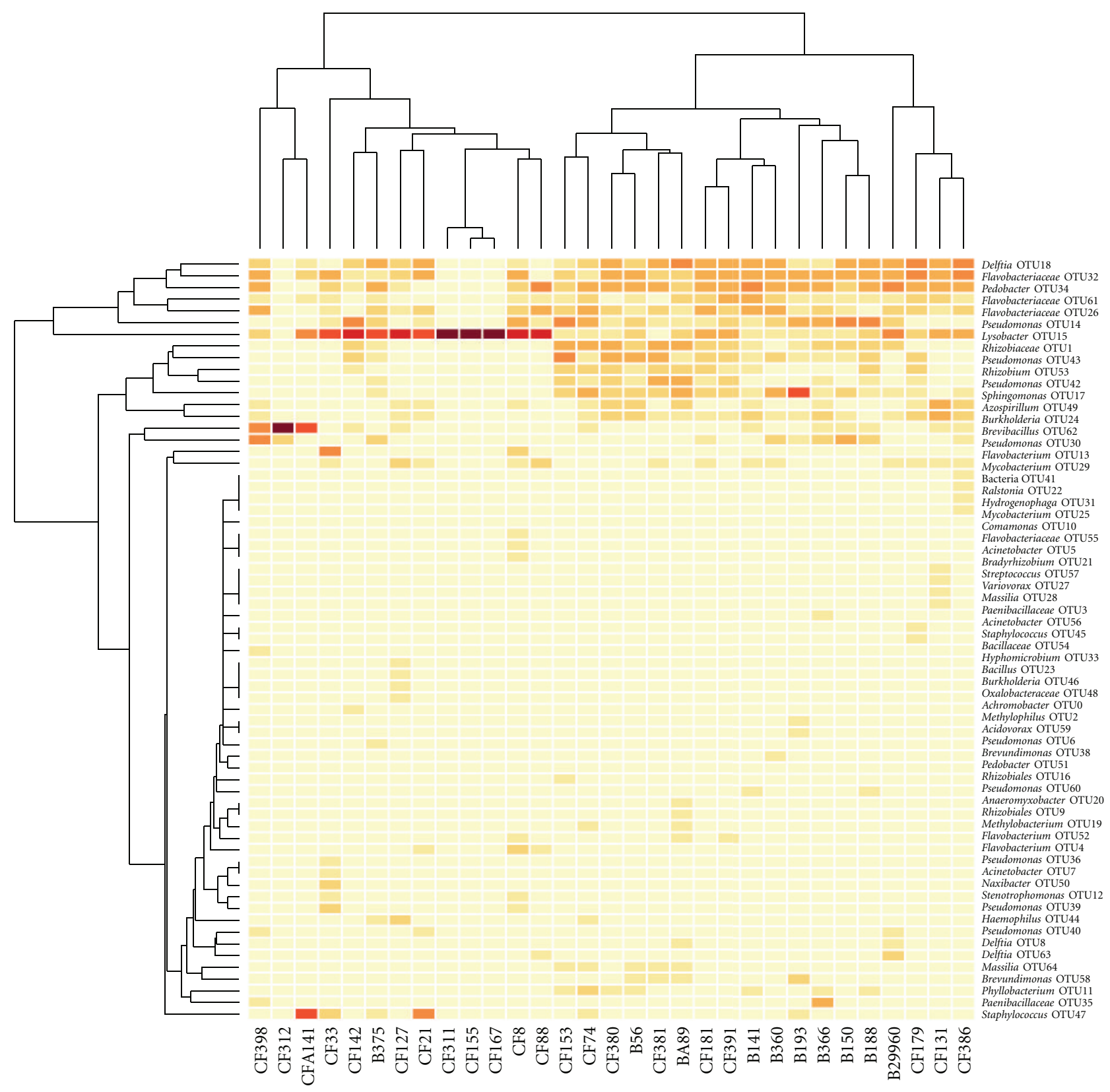

FIGURE 2: Heatmap showing clustering of bacterial communities and similarities in operational taxonomic unit (OTU) distribution among patients. Sample numbers are listed horizontally along the bottom; "B" indicates bronchiectasis samples, and "CF" indicates CF samples. OTUs and their taxonomic classifications are listed vertically on the right. Darker colors indicate higher abundance; lighter colors indicate low abundance or absence of a particular OTU.

and using these distances to infer a UPGMA phylogenetic tree (Figure 3). As is clear from the grouping of different patients in the tree, there exist no clear differences between bacterial communities from bronchiectasis and CF patients. In fact, when sequence divergence is taken into account, the small cluster of bronchiectasis patients noted in Figure 2 is no longer present (Figure 3).

Finally, we used principal components analysis (PCA) to identify whether differences exist between bronchiectasis and CF communities. PCA is useful for removing correlations in the data that may obscure the detection of differences that exist due to a diagnosis of bronchiectasis versus CF (e.g., age of patient). The results from PCA agree with the results from the other two methods described above, namely, that significant differences in bacterial community structure could not be found when comparing bronchiectasis to $\mathrm{CF}$ patients (Figure 4).

\section{Discussion}

Bacterial infection is a major cause of morbidity in individuals with $\mathrm{CF}$ and non-CF bronchiectasis. Management 
TABLE 1: Sequences and OTUs for each patient.

\begin{tabular}{|c|c|c|c|c|}
\hline Patient & Source* & $\begin{array}{c}\text { Number of } \\
\text { rRNA } \\
\text { sequences }\end{array}$ & $\begin{array}{c}\text { Number of OTUs } \\
\text { (number of } \\
\text { Pseudomonas OTUs) }\end{array}$ & $\begin{array}{l}\text { Number } \\
\text { of genera }\end{array}$ \\
\hline A141 & $\mathrm{CF}$ & 39 & $6(1)$ & 5 \\
\hline A89 & B & 81 & $21(3)$ & 12 \\
\hline 8 & CF & 54 & $17(3)^{+}$ & 8 \\
\hline 21 & CF & 38 & $11(2)^{+}$ & 6 \\
\hline 33 & CF & 81 & $14(4)^{+}$ & 8 \\
\hline 56 & B & 101 & $21(3)$ & 11 \\
\hline 74 & $\mathrm{CF}$ & 79 & $20(3)$ & 13 \\
\hline 88 & $\mathrm{CF}$ & 59 & $8(1)^{+}$ & 4 \\
\hline 127 & CF & 86 & $14(1)^{+}$ & 8 \\
\hline 131 & CF & 89 & $14(1)^{+}$ & 11 \\
\hline 141 & B & 98 & $17(4)$ & 9 \\
\hline 142 & CF & 87 & $10(2)^{+}$ & 5 \\
\hline 150 & B & 90 & $13(2)$ & 7 \\
\hline 153 & $\mathrm{CF}$ & 81 & $15(3)$ & 7 \\
\hline 155 & CF & 28 & $1(1)^{+}$ & 1 \\
\hline 167 & CF & 43 & $1(1)^{+}$ & 1 \\
\hline 179 & CF & 63 & $14(3)$ & 9 \\
\hline 181 & $\mathrm{CF}$ & 85 & $15(3)^{+}$ & 8 \\
\hline 188 & B & 86 & $15(4)$ & 8 \\
\hline 193 & B & 77 & $16(2)^{+}$ & 10 \\
\hline 311 & $\mathrm{CF}$ & 18 & $1(1)^{+}$ & 1 \\
\hline 312 & CF & 27 & $2(0)$ & 2 \\
\hline 360 & B & 86 & $13(2)$ & 7 \\
\hline 366 & B & 77 & $18(2)$ & 10 \\
\hline 375 & B & 84 & $15(3)^{+}$ & 8 \\
\hline 380 & CF & 91 & $15(3)$ & 8 \\
\hline 381 & CF & 94 & $19(3)$ & 11 \\
\hline 386 & $\mathrm{CF}$ & 70 & $16(2)$ & 11 \\
\hline 391 & CF & 79 & $14(3)$ & 7 \\
\hline 398 & CF & 76 & $13(2)$ & 8 \\
\hline 29960 & B & 175 & $18(4)$ & 9 \\
\hline
\end{tabular}

${ }^{*}$ CF denotes cystic fibrosis patients; B denotes bronchiectasis. rRNA: ribosomal ribonucleic acid; OTU: operational taxonomic unit.

${ }^{+}$Pseudomonas is the numerically predominant taxon.

Table 2: Phyla detected in lungs.

\begin{tabular}{lcc}
\hline Phylum & $\begin{array}{c}\text { \% sequences in CF } \\
\text { patients }\end{array}$ & $\begin{array}{c}\text { \% sequences in bronchiectasis } \\
\text { patients }\end{array}$ \\
\hline Proteobacteria & 57.3 & 64.6 \\
Bacteroidetes & 37.6 & 30.2 \\
Actinobacteria & 3.5 & 0.1 \\
Firmicutes & 1.6 & 5.1 \\
\hline
\end{tabular}

of bacterial infections historically has been based primarily on culture-based isolation of a limited number of species considered pathogenic, but there is growing appreciation of the polymicrobial nature of pulmonary infections particularly in the setting of chronic illnesses such as CF,

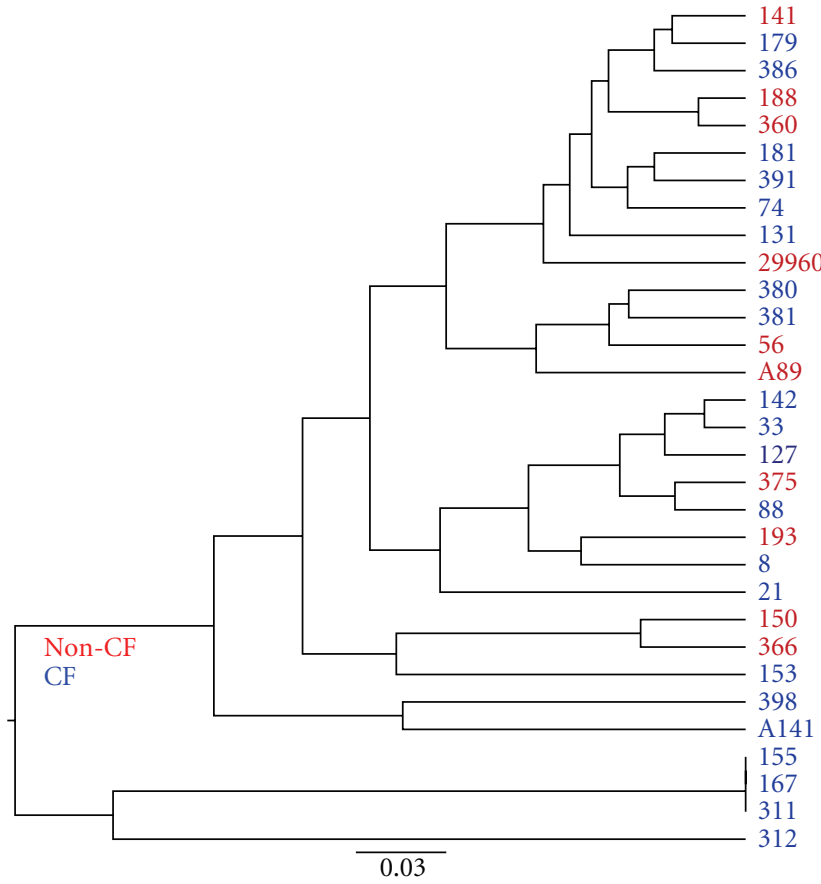

FIGURE 3: Unweighted pair group method with arithmetic mean (UPGMA) tree inferred using UniFrac distances. Red and blue names indicate bronchiectasis and CF, respectively.

and increasing evidence indicates that interactions between species may alter virulence properties or antibiotic susceptibility of individual species [33]. Better understanding of such interactions and their potential clinical impacts requires, as a first step, more complete characterization of the composition of the microbial community of the lungs. We describe here the application of culture-independent metagenomicbased strategies to the study of the bacterial communities of the lung. While a number of studies have applied similar strategies to the study of respiratory microbiota in CF patients [17-27], this study is, to our knowledge, the first reporting culture-independent sequence-based profiling of bacterial species directly from lung tissue of patients with non-CF bronchiectasis. Our data demonstrate significant diversity in the pulmonary bacterial communities of both $\mathrm{CF}$ and bronchiectasis patients but did not detect significant differences in overall community composition between these two groups of patients.

Proteobacteria predominated in both $\mathrm{CF}$ and non-CF groups (Tables 2 and 3), with Pseudomonas being the most prevalent genus overall. Pseudomonas was numerically predominant in 2 of 10 patients with bronchiectasis, which appears to be in keeping with previously reported rates of isolation of $P$. aeruginosa from bronchiectasis patients in the neighborhood of $18 \%$ [4]. Interestingly, however, while Pseudomonas was numerically predominant in 2 bronchiectasis patients, it was identified as a minor constituent of the bacterial community in the remaining 8 bronchiectasis patients. On the other hand, whereas $H$. influenzae is typically cultured from approximately $40 \%$ of bronchiectasis 
TABLE 3: Taxonomic classification of all sequences.

\begin{tabular}{|c|c|c|c|}
\hline Taxonomy & Level of resolution & Phylum & Total sequences \\
\hline Pseudomonas OTU14 & Genus & Proteobacteria & 490 \\
\hline Delftia OTU18 & Genus & Proteobacteria & 260 \\
\hline Flavobacteriaceae OTU32 & Family & Bacteroidetes & 227 \\
\hline Delftia OTU37 & Genus & Proteobacteria & 207 \\
\hline Flavobacteriaceae OTU26 & Family & Bacteroidetes & 148 \\
\hline Flavobacteriaceae OTU61 & Family & Bacteroidetes & 118 \\
\hline Pseudomonas OTU42 & Genus & Proteobacteria & 110 \\
\hline Rhizobiaceae OTU1 & Family & Proteobacteria & 102 \\
\hline Lysobacter OTU15 & Genus & Proteobacteria & 98 \\
\hline Burkholderia OTU24 & Genus & Proteobacteria & 72 \\
\hline Pedobacter OTU34 & Genus & Bacteroidetes & 70 \\
\hline Brevibacillus OTU62 & Genus & Firmicutes & 66 \\
\hline Azospirillum OTU49 & Genus & Proteobacteria & 63 \\
\hline Rhizobium OTU53 & Genus & Proteobacteria & 45 \\
\hline Pseudomonas OTU43 & Genus & Proteobacteria & 42 \\
\hline Sphingomonas OTU17 & Genus & Proteobacteria & 36 \\
\hline Staphylococcus OTU47 & Genus & Firmicutes & 25 \\
\hline Pseudomonas OTU30 & Genus & Proteobacteria & 23 \\
\hline Flavobacterium OTU13 & Genus & Bacteroidetes & 20 \\
\hline Phyllobacterium OTU11 & Genus & Proteobacteria & 14 \\
\hline Brevundimonas OTU58 & Genus & Proteobacteria & 10 \\
\hline Massilia OTU64 & Genus & Proteobacteria & 10 \\
\hline Paenibacillaceae OTU35 & Family & Firmicutes & 9 \\
\hline Delftia OTU63 & Genus & Proteobacteria & 7 \\
\hline Haemophilus OTU44 & Genus & Proteobacteria & 6 \\
\hline Pseudomonas OTU39 & Genus & Proteobacteria & 6 \\
\hline Delftia OTU8 & Genus & Proteobacteria & 4 \\
\hline Flavobacterium OTU4 & Genus & Bacteroidetes & 4 \\
\hline Pseudomonas OTU40 & Genus & Proteobacteria & 4 \\
\hline Flavobacterium OTU52 & Genus & Bacteroidetes & 3 \\
\hline Naxibacter OTU50 & Genus & Proteobacteria & 3 \\
\hline Stenotrophomonas OTU12 & Genus & Proteobacteria & 3 \\
\hline Brevundimonas OTU38 & Genus & Proteobacteria & 2 \\
\hline Methylobacterium OTU19 & Genus & Proteobacteria & 2 \\
\hline Pseudomonas OTU60 & Genus & Proteobacteria & 2 \\
\hline Rhizobiales OTU16 & Order & Proteobacteria & 2 \\
\hline Achromobacter OTU0 & Genus & Proteobacteria & 1 \\
\hline Acidovorax OTU59 & Genus & Proteobacteria & 1 \\
\hline Acinetobacter OTU5 & Genus & Proteobacteria & 1 \\
\hline Acinetobacter OTU56 & Genus & Proteobacteria & 1 \\
\hline Acinetobacter OTU7 & Genus & Proteobacteria & 1 \\
\hline Anaeromyxobacter OTU20 & Genus & Proteobacteria & 1 \\
\hline Bacillaceae OTU54 & Family & Firmicutes & 1 \\
\hline Bacillus OTU23 & Genus & Firmicutes & 1 \\
\hline Bacteria OTU41 & Domain & Bacteria & 1 \\
\hline Bradyrhizobium OTU21 & Genus & Proteobacteria & 1 \\
\hline Burkholderia OTU46 & Genus & Proteobacteria & 1 \\
\hline Comamonas OTU10 & Genus & Proteobacteria & 1 \\
\hline Flavobacteriaceae OTU55 & Family & Bacteroidetes & 1 \\
\hline Hydrogenophaga OTU31 & Genus & Proteobacteria & 1 \\
\hline
\end{tabular}


TABle 3: Continued.

\begin{tabular}{lccc}
\hline Taxonomy & Level of resolution & Phylum & Total sequences \\
\hline Hyphomicrobium OTU33 & Genus & Proteobacteria & 1 \\
Massilia OTU28 & Genus & Proteobacteria & 1 \\
Methylophilus OTU2 & Genus & Proteobacteria & Actinobacteria \\
Mycobacterium OTU25 & Genus & Actinobacteria & 1 \\
Mycobacterium OTU29 & Genus & Proteobacteria & 1 \\
Oxalobacteraceae OTU48 & Family & Firmicutes & 1 \\
Paenibacillaceae OTU3 & Family & Bacteroidetes & 1 \\
Pedobacter OTU51 & Genus & Proteobacteria \\
Pseudomonas OTU36 & Genus & Proteobacteria & 1 \\
Pseudomonas OTU6 & Genus & Proteobacteria \\
Ralstonia OTU22 & Genus & Proteobacteria \\
Rhizobiales OTU9 & Order & Firmicutes \\
Staphylococcus OTU45 & Genus & Firmicutes \\
Streptococcus OTU57 & Genus & Proteobacteria \\
Variovorax OTU27 & Genus & & 1 \\
\hline OUT: op & & 1
\end{tabular}

OUT: operational taxonomic unit.

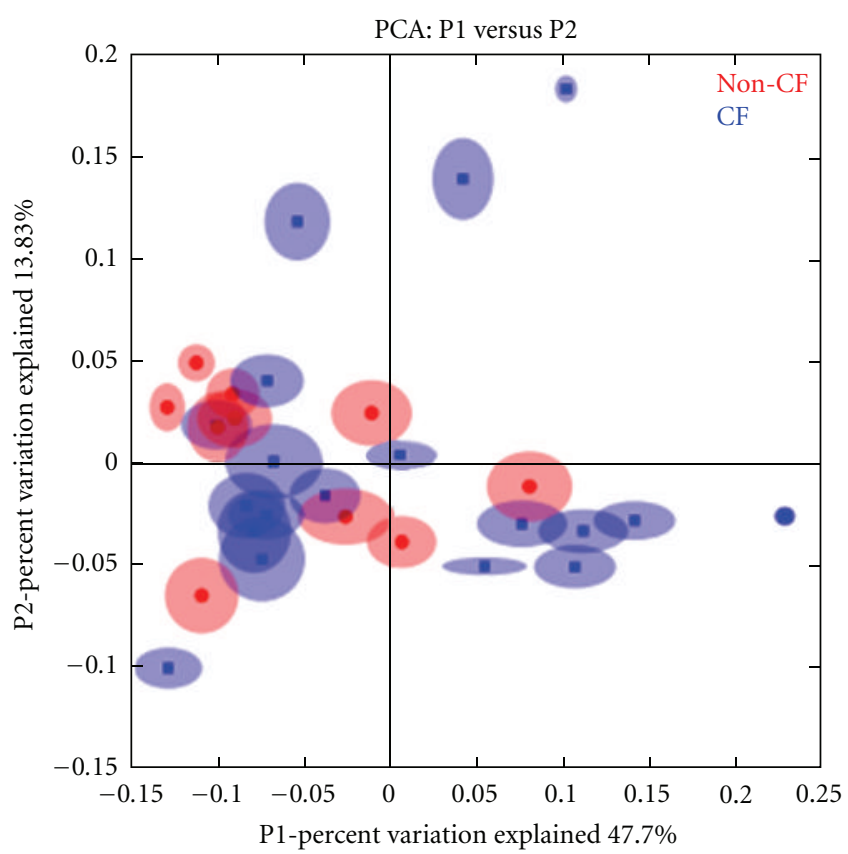

Figure 4: Principal components analysis (PCA) of bronchiectasis (red) and CF patients (blue).

patients, it was detected in only 1 of 10 bronchiectasis patients in this study. The reasons for these discrepancies between our culture-independent data and previous culturebased studies may be several-fold. Given that the present study examined tissue samples from resected lung specimens including many from patients undergoing lung transplantation, the bacterial community profiles generated here most likely represent those present in end-stage lung disease, and, together with the relatively small number of specimens profiled here, therefore may not be completely representative of the microflora in earlier stages of disease process. Further, this study examined lung tissue samples, whereas previous studies of bacterial species in bronchiectasis were based largely on cultures of sputum samples. Because $H$. influenzae frequently colonizes the upper respiratory tract, it may be the case that previous sputum-based studies have overestimated the prevalence of $H$. influenzae in the lung itself. Finally, the high frequency of Pseudomonas detection in our bronchiectasis data set compared to culture-based studies could possibly be due to inhibition of growth of Pseudomonas in culture by interspecies interactions with other, more prevalent species present within sputum samples. Such inhibition could possibly account for at least part of the large proportion of sputum samples from bronchiectasis patients in which no pathogens are detected by conventional culture methods [2]. Similar factors could also account for our detection of Burkholderia in 4 of 10 bronchiectasis patients, whereas a previous culture-based study failed to isolate any Burkholderia from sputum samples of patients with bronchiectasis [13].

Comparison of pulmonary bacterial community profiles from bronchiectasis patients with those obtained from $\mathrm{CF}$ patients using three different methods found no significant differences in community structure between the two groups. At this point, it is uncertain whether this is simply a function of the fact that the specimens analyzed in this study were derived from end-stage lungs (both $\mathrm{CF}$ and non-CF) and whether the pulmonary bacterial communities of CF and bronchiectasis patients at earlier stages may demonstrate more significant differences. Longitudinal studies profiling respiratory samples from bronchiectasis patients in comparison with CF patients over time are needed to shed light on this question.

In summary, our data indicate significant diversity in the bacterial community of the lungs in both CF and non-CF bronchiectasis patients but did not detect significant differences in the bacterial community structure 
between these two groups. The application of cultureindependent methods, such as those described here, in concert with conventional culture-based studies should help significantly advance understanding of the microbiology of the bronchiectatic airway.

\section{Acknowledgments}

The authors thank Paul Chartrand and Jimmy Chen for technical assistance and Sassan Azad for assistance with data collection. This study was funded in part by the Ontario Thoracic Society, the J. P. Bickell Foundation, the National Sanitarium Association, the Canadian Institutes of Health Research (CIHR) (CHM-94308), and the Physicians of Ontario through the Physicians' Services Incorporated Foundation. Dr. Guttman is supported by grants from the Natural Sciences and Engineering Research Council of Canada, CIHR, and the Canada Research Chairs program. Dr. Maughan is recipient of a fellowship from Cystic Fibrosis Canada.

\section{References}

[1] P. King, S. Holdsworth, N. Freezer, and P. Holmes, "Bronchiectasis," Internal Medicine Journal, vol. 36, no. 11, pp. 729-737, 2006.

[2] P. T. King, "The pathophysiology of bronchiectasis," International Journal of Chronic Obstructive Pulmonary Disease, vol. 4, pp. 411-419, 2009.

[3] P. T. King, S. R. Holdsworth, N. J. Freezer, E. Villanueva, M. Gallagher, and P. W. Holmes, "Outcome in adult bronchiectasis," Journal of Chronic Obstructive Pulmonary Disease, vol. 2, no. 1, pp. 27-34, 2005.

[4] P. T. King, S. R. Holdsworth, N. J. Freezer, E. Villanueva, and P. W. Holmes, "Microbiologic follow-up study in adult bronchiectasis," Respiratory Medicine, vol. 101, no. 8, pp. 1633-1638, 2007.

[5] M. C. Pasteur, S. M. Helliwell, S. J. Houghton et al., "An investigation into causative factors in patients with bronchiectasis," American Journal of Respiratory and Critical Care Medicine, vol. 162, no. 4, pp. 1277-1284, 2000.

[6] J. Angrill, C. Agustí, R. De Celis et al., "Bronchial inflammation and colonization in patients with clinically stable bronchiectasis," American Journal of Respiratory and Critical Care Medicine, vol. 164, no. 9, pp. 1628-1632, 2001.

[7] J. Angrill, C. Agustí, R. De Celis et al., "Bacterial colonisation in patients with bronchiectasis: microbiological pattern and risk factors," Thorax, vol. 57, no. 1, pp. 15-19, 2002.

[8] L. V. M. Möller, W. Timens, W. Van Der Bij et al., "Haemophilus influenzae in lung explants of patients with end-stage pulmonary disease," American Journal of Respiratory and Critical Care Medicine, vol. 157, no. 3, pp. 950-956, 1998.

[9] H. Cabello, A. Torres, R. Celis et al., "Bacterial colonization of distal airways in healthy subjects and chronic lung disease: a bronchoscopic study," European Respiratory Journal, vol. 10, no. 5, pp. 1137-1144, 1997.

[10] M. B. Nicotra, M. Rivera, A. M. Dale, R. Shepherd, and R. Carter, "Clinical, pathophysiologic, and microbiologic characterization of bronchiectasis in an aging cohort," Chest, vol. 108, no. 4, pp. 955-961, 1995.

[11] J. A. Pang, A. Cheng, H. S. Chan, and G. French, "The bacteriology of bronchiectasis in Hong Kong investigated by protected catheter brush and bronchoalveolar lavage," American Review of Respiratory Disease, vol. 139, no. 1, pp. 1417, 1989. pt

[12] R. L. Gibson, J. L. Burns, and B. W. Ramsey, "Pathophysiology and management of pulmonary infections in cystic fibrosis," American Journal of Respiratory and Critical Care Medicine, vol. 168, no. 8, pp. 918-951, 2003.

[13] J. Hyde and H. Humphreys, "Absence of Burkholderia cepacia from the respiratory tract of non-cystic fibrosis patients," European Journal of Clinical Microbiology \& Infectious Diseases, vol. 16, no. 3, pp. 253-254, 1997.

[14] S. G. Tringe and E. M. Rubin, "Metagenomics: DNA sequencing of environmental samples," Nature Reviews Genetics, vol. 6, no. 11, pp. 805-814, 2005.

[15] P. J. Turnbaugh, R. E. Ley, M. Hamady, C. M. Fraser-Liggett, R. Knight, and J. I. Gordon, "The human microbiome project," Nature, vol. 449, no. 7164, pp. 804-810, 2007.

[16] A. Mullard, "Microbiology: the inside story," Nature, vol. 453, no. 7195, pp. 578-580, 2008.

[17] G. B. Rogers, M. P. Carroll, D. J. Serisier et al., "Use of $16 \mathrm{~S}$ rRNA gene profiling by terminal restriction fragment length polymorphism analysis to compare bacterial communities in sputum and mouthwash samples from patients with cystic fibrosis," Journal of Clinical Microbiology, vol. 44, no. 7, pp. 2601-2604, 2006.

[18] G. B. Rogers, M. P. Carroll, D. J. Serisier et al., "Bacterial activity in cystic fibrosis lung infections," Respiratory Research, vol. 6, article 49, 2005.

[19] G. B. Rogers, M. P. Carroll, D. J. Serisier, P. M. Hockey, G. Jones, and K. D. Bruce, "Characterization of bacterial community diversity in cystic fibrosis lung infections by use of $16 \mathrm{~S}$ ribosomal DNA terminal restriction fragment length polymorphism profiling," Journal of Clinical Microbiology, vol. 42, no. 11, pp. 5176-5183, 2004.

[20] G. B. Rogers, C. A. Hart, J. R. Mason, M. Hughes, M. J. Walshaw, and K. D. Bruce, "Bacterial diversity in cases of lung infection in cystic fibrosis patients: $16 \mathrm{~S}$ ribosomal DNA (rDNA) length heterogeneity PCR and 16S rDNA terminal restriction fragment length polymorphism profiling," Journal of Clinical Microbiology, vol. 41, no. 8, pp. 3548-3558, 2003.

[21] M. Kolak, F. Karpati, H. J. Monstein, and J. Jonasson, "Molecular typing of the bacterial flora in sputum of cystic fibrosis patients," International Journal of Medical Microbiology, vol. 293, no. 4, pp. 309-317, 2003.

[22] J. K. Harris, M. A. De Groote, S. D. Sagel et al., "Molecular identification of bacteria in bronchoalveolar lavage fluid from children with cystic fibrosis," Proceedings of the National Academy of Sciences of the United States of America, vol. 104, no. 51, pp. 20529-20533, 2007.

[23] F. Bittar, H. Richet, J. C. Dubus et al., "Molecular detection of multiple emerging pathogens in sputa from cystic fibrosis patients," PLoS ONE, vol. 3, no. 8, article e2908, 2008.

[24] C. D. Sibley, M. D. Parkins, H. R. Rabin, K. Duan, J. C. Norgaard, and M. G. Surette, "A polymicrobial perspective of pulmonary infections exposes an enigmatic pathogen in cystic fibrosis patients," Proceedings of the National Academy of Sciences of the United States of America, vol. 105, no. 39, pp. 15070-15075, 2008.

[25] M. Doud, E. Zeng, L. Schneper, G. Narasimhan, and K. Mathee, "Approaches to analyse dynamic microbial communities such as those seen in cystic fibrosis lung," Human Genomics, vol. 3, no. 3, pp. 246-256, 2009. 
[26] M. J. Cox, M. Allgaier, B. Taylor et al., "Airway microbiota and pathogen abundance in age-stratified cystic fibrosis patients," PLoS ONE, vol. 5, no. 6, article e11044, 2010.

[27] A. M. Guss, G. Roeselers, I. L. G. Newton et al., "Phylogenetic and metabolic diversity of bacteria associated with cystic fibrosis," ISME Journal, vol. 5, no. 1, pp. 20-29, 2011.

[28] S. F. Altschul, W. Gish, W. Miller, E. W. Myers, and D. J. Lipman, "Basic local alignment search tool," Journal of Molecular Biology, vol. 215, no. 3, pp. 403-410, 1990.

[29] J. G. Caporaso, J. Kuczynski, J. Stombaugh et al., "QIIME allows analysis of high-throughput community sequencing data," Nature Methods, vol. 7, no. 5, pp. 335-336, 2010.

[30] Q. Wang, G. M. Garrity, J. M. Tiedje, and J. R. Cole, "Naïve Bayesian classifier for rapid assignment of rRNA sequences into the new bacterial taxonomy," Applied and Environmental Microbiology, vol. 73, no. 16, pp. 5261-5267, 2007.

[31] C. Lozupone and R. Knight, "UniFrac: a new phylogenetic method for comparing microbial communities," Applied and Environmental Microbiology, vol. 71, no. 12, pp. 8228-8235, 2005.

[32] R Development Core Team, R: A Language and Environment for Statistical Computing, R Foundation for Statistical Computing, Vienna, Austria, 2011.

[33] C. D. Sibley, K. Duan, C. Fischer et al., "Discerning the complexity of community interactions using a Drosophila model of polymicrobial infections," PLoS Pathogens, vol. 4, no. 10, Article ID e1000184, 2008. 


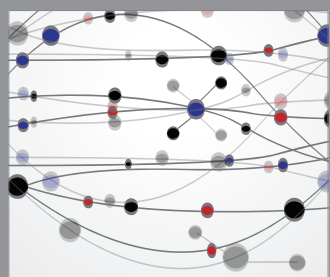

The Scientific World Journal
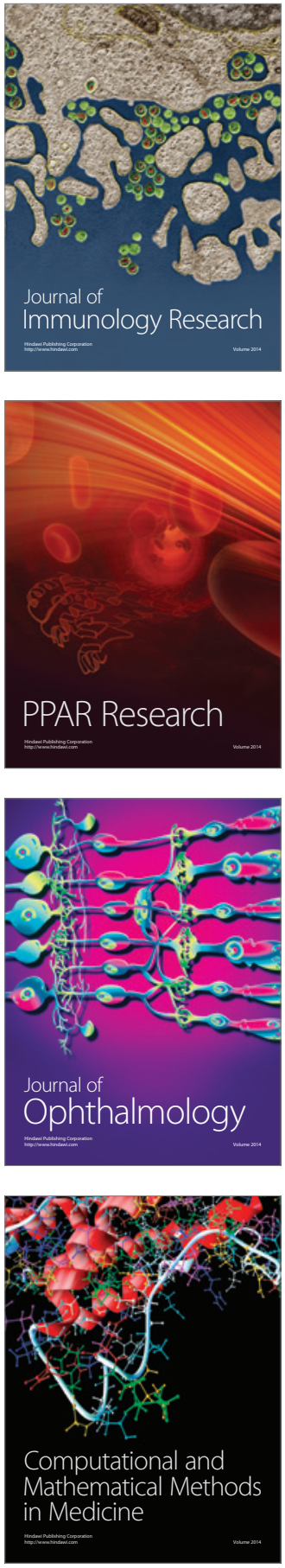

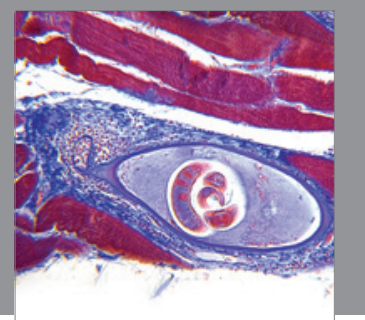

Gastroenterology

Research and Practice
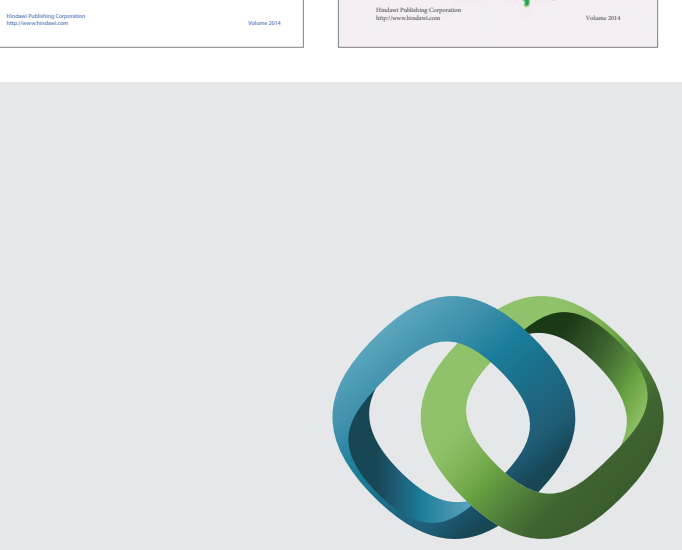

\section{Hindawi}

Submit your manuscripts at

http://www.hindawi.com
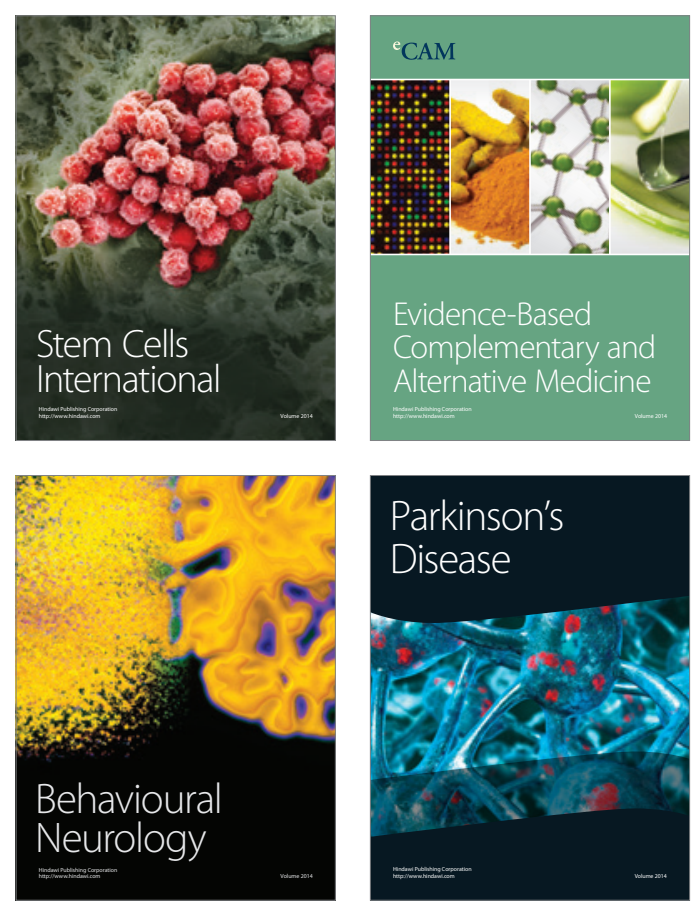

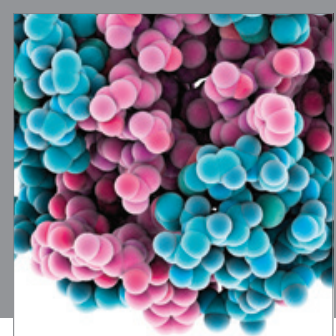

Journal of
Diabetes Research

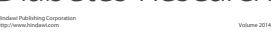

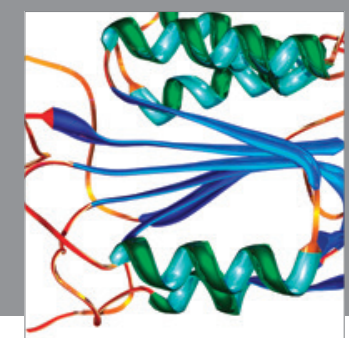

Disease Markers
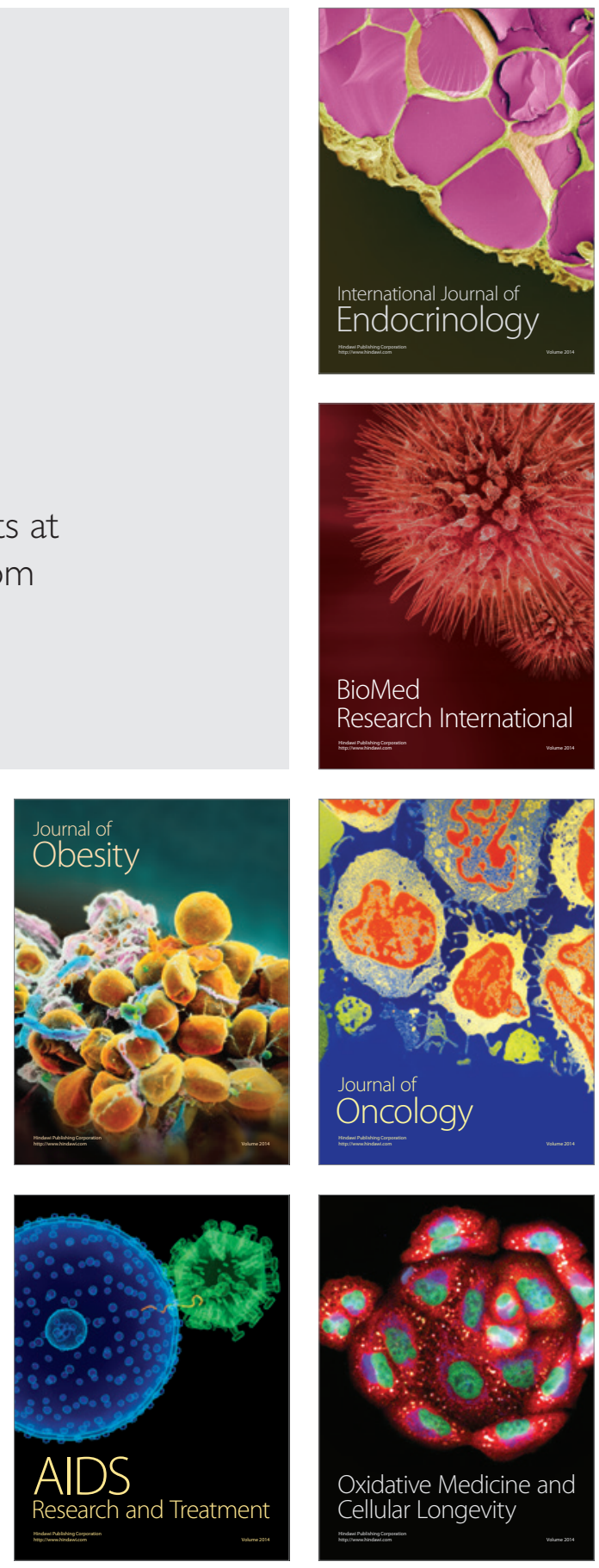